\title{
Vegetable intake in Korea: data from the Korean National Health and Nutrition Examination Survey 1998, 2001 and 2005
}

\author{
Jung-Sug Lee and Jeongseon Kim* \\ Cancer Epidemiology Branch, Research Institute, National Cancer Center, 111 Jungbalsan-ro, Madu-1-dong, Ilsandong-gu, \\ Goyang-si, Gyeoggi-do 410-769, South Korea
}

(Received 24 March 2009 - Revised 17 November 2009 - Accepted 19 November 2009 - First published online 4 February 2010)

The purpose of the present study was to analyse vegetable intake by the Korean population based on different meal formats. Twenty-four-hour dietary recall data from the Korean National Health and Nutrition Examination Survey were used to assess daily vegetable intake and consumption ratio of vegetables for different meal types/dining locations. Analysis was stratified by sex and age group. Daily vegetable intake increased from $293.5 \mathrm{~g}$ in 1998 to $305.7 \mathrm{~g}$ in 2001 and to $335.9 \mathrm{~g}$ in 2005 . Findings were similar in men and women, as well as in each age group. Analyses for each meal type revealed that vegetable intake during breakfast did not substantially change, but vegetable intake increased during lunch and dinner. While vegetable consumption ratio during breakfast decreased, it increased during lunch and snack time. Men and women showed similar change in the analysis for meal types and dining locations. There were some differences between the age groups in daily vegetable intake depending on the meal type. Another notable observation was the increasing change of vegetable intake during lunch and during meals eaten at cafeterias and restaurants in all the age groups. Analysis of vegetable consumption ratio showed an increased consumption in cafeterias, restaurants, and other places and decreased vegetable intake at home for those aged 16-64 years with time. Since this change may be related to improvements in socioeconomic status, it will be necessary to further investigate difference in vegetable intake based on socioeconomic levels within the population.

Vegetable intake: Korean National Health and Nutrition Examination Survey: Korea

A vegetable-rich diet is thought to be beneficial to health and, if adopted by more of the population, may be important in reducing levels of weight gain $^{(1)}$, heart disease ${ }^{(2-4)}$, cancer $^{(5,6)}$ and other chronic diseases ${ }^{(7,8)}$. There have been a variety of studies that have measured the association between trends in vegetable intake and its effects on disease prevalence. More recently, a survey investigating trends in vegetable intake by the US population revealed that the mean frequency of daily vegetable consumption declined slightly from 1994 through 2005 (Standardised change: -0.11 servings $/ \mathrm{d})^{(9)}$. A study on trends in food consumption in Spain reported that vegetable intake decreased between 1992 and $2003(-18.7 \mathrm{~g} / \mathrm{d}$ for $\mathrm{men} ;-16.5 \mathrm{~g} / \mathrm{d}$ for women $)^{(10)}$. On the other hand, another study that assessed trends in food intake in Portugal showed that the percentage of subjects consuming vegetables increased from 1987 through 1999 (men, 70.7-80.8\%; women, 70.7-82.9\%) ${ }^{(11)}$.

The WHO and the World Cancer Research Fund/American Institute for Cancer Research recommend a daily intake of at least $400 \mathrm{~g}$ or $14 \mathrm{oz}$ of fruits and non-starch vegetables; this recommendation has been translated into public health advice to eat five or more portions of fruits and non-starch vegetables per day ${ }^{(12,13)}$. Beginning in 1985, the United States Department of Agriculture, in their report Dietary Guidelines for Healthy Americans, recommended the consumption of at least two servings of fruits and three servings of vegetables daily ${ }^{(14)}$. In 2005 , the report Japanese Food Guide Spinning Top selected by the Ministry of Health, Labour and Welfare and the Ministry of Agriculture, Forestry and Fisheries in Japan recommended the consumption of five to six servings of vegetables daily ${ }^{(15)}$. In Korea, the Korean Nutrition Society created the Dietary Reference Intakes for Koreans in 2005, recommending a daily consumption of five to six servings of vegetables by adults $^{(16)}$. The objectives of the Public Health Plan 2010 for Korea include increasing the percentage of Koreans, who eat sufficient vegetables daily ${ }^{(17)}$. Assessing the vegetable consumption change is important for tracking the effectiveness of public health initiatives in meeting this goal and for planning future objectives. However, vegetable intake analyses do not examine differences in consumption in terms of meal formats (meal types and dining locations). The purpose of the present study is to analyse difference in vegetable intake (1998-2005) by the Korean population for different meal formats using the Korean National Health and Examination Survey (KNHANES).

\section{Subjects and methods}

The data used in the present study were derived from KNHANES 1998, 2001 and 2005. KNHANES are noninstitutional, Korean civilian population surveys that were 
conducted in 1998, 2001 and 2005. The surveys were designed based on stratified multistage samplings from the South Korean population in different geographic areas and in different age and sex groups. Trained interviewers conducted surveys in households and administered a structured questionnaire to obtain information on sociodemography, lifestyle, health characteristics and nutritional status including dietary supplement use for each respondent.

The study included 8998 subjects ( $\geq 7$ years of age) in 1998, 8689 subjects in 2001 and 7910 subjects in 2005 with complete demographic and dietary data. As part of the standard KNHANES data collection protocol, 24-hour dietary recalls were elicited and were used to estimate daily vegetable consumption. Foods were coded and analysed using a five-digit food coding scheme developed by the Ministry of Health and Welfare in 1998, 2001 and 2005 for the KNHANES. Food items were categorised by food group and subgroup. Items falling under the category of 'vegetable' were further characterised as leafy dark green vegetables, green and yellow vegetables, tomatoes, green beans, root vegetables, white vegetables and kimchies.

Meal types were categorised based on meal time (breakfast, lunch, dinner or snack) and dining locations were categorised by places of dining: home (own home or neighbour/relative's home); cafeteria (school, work place, nursery/kindergarten, senior's house, dining place for college-aged students or welfare centre); restaurant (street food stand, convenience store, bakery or fast food); others.

All the analyses were conducted using a survey weighting to account for the complex survey design, which consisted of multistage, stratified, clustered samples. Probability sampling weights were used in conjunction with strata and primary sampling units to apply weight to the data analysis. Subject characteristics were compared between survey periods using a $\chi^{2}$ test. Mean values with their standard errors of vegetable intake for each meal type and dining location were calculated. Bonferroni's multiple $t$ test was used for verification of significance for each survey period. All statistical analyses were performed using SURVEY PROCEDURE of Statistical Analysis System software (version 9.12; SAS Institute, Cary, NC, USA) and DESCRIPT PROCEDURE of SUDAN software (release 9.0, Research Triangle Institute, Research Triangle Park, NC, USA) applying a significance level of $P<0 \cdot 05$.

\section{Results}

\section{Subjects}

General data about the subjects surveyed are shown in Table 1. The number of female subjects was greater than the number of male subjects in all years of the survey, but the number of female subjects in 2005 was lower than that in 1998 $(P<0.001)$. The largest participant group in all the years was the 30-49 age group, making up more than $36 \%$ of those surveyed. The largest group of participants consisted of those residing in large cities, accounting for more than $47 \%$ of total participants surveyed in 1998, 2001 and 2005. Levels of income and education were higher in 2005 compared with those in 1998; that is, while the proportions of subjects from low and middle income groups decreased, the proportion of high income subjects increased. Subjects with middle school or lower education decreased from $43.9 \%$ in 1998 to $37.2 \%$ in 2005, whereas those with college or higher education increased from $20.3 \%$ in 1998 to $29.7 \%$ in $2005(P<0 \cdot 001)$

Table 1. General subject characteristics

(Number of subjects and percentages)

\begin{tabular}{|c|c|c|c|c|c|c|c|}
\hline \multirow{2}{*}{ Year... } & \multicolumn{2}{|c|}{1998} & \multicolumn{2}{|c|}{2001} & \multicolumn{2}{|c|}{2005} & \multirow[b]{2}{*}{$P$} \\
\hline & $n$ & $\%$ & $n$ & $\%$ & $n$ & $\%$ & \\
\hline \multicolumn{8}{|l|}{ Sex } \\
\hline Male & 4252 & $48 \cdot 0$ & 4094 & $46 \cdot 7$ & 3627 & $49 \cdot 7$ & $<0.001$ \\
\hline Female & 4746 & $52 \cdot 0$ & 4595 & 53.3 & 4283 & $50 \cdot 3$ & \\
\hline \multicolumn{8}{|l|}{ Age (years) } \\
\hline $7-12$ & 887 & $10 \cdot 2$ & 1060 & $11 \cdot 7$ & 905 & $10 \cdot 0$ & $<0.001$ \\
\hline $13-15$ & 426 & $5 \cdot 1$ & 464 & $5 \cdot 3$ & 429 & $5 \cdot 1$ & \\
\hline $16-18$ & 504 & $5 \cdot 8$ & 360 & $4 \cdot 3$ & 299 & $3 \cdot 7$ & \\
\hline $19-29$ & 1485 & $18 \cdot 3$ & 1316 & 15.5 & 1022 & $18 \cdot 3$ & \\
\hline $30-49$ & 3174 & 36.9 & 3247 & 36.9 & 2887 & $37 \cdot 2$ & \\
\hline $50-64$ & 1577 & 15.5 & 1353 & $16 \cdot 3$ & 1396 & $15 \cdot 8$ & \\
\hline$\geq 65$ & 945 & $8 \cdot 2$ & 889 & $9 \cdot 9$ & 972 & $9 \cdot 9$ & \\
\hline \multicolumn{8}{|l|}{ Residential area } \\
\hline Large city & 3596 & $48 \cdot 3$ & 4044 & $50 \cdot 4$ & 3632 & $47 \cdot 2$ & 0.759 \\
\hline Small city & 2223 & 31.4 & 2807 & $31 \cdot 7$ & 2705 & $35 \cdot 1$ & \\
\hline Rural area & 3179 & $20 \cdot 4$ & 1838 & $20 \cdot 4$ & 1573 & $17 \cdot 7$ & \\
\hline \multicolumn{8}{|l|}{ Income* } \\
\hline Low & 3794 & 37.9 & 2222 & $25 \cdot 8$ & 1843 & $21 \cdot 7$ & $<0.001$ \\
\hline Middle & 3680 & 43.2 & 3647 & 41.5 & 2978 & 37.9 & \\
\hline High & 1524 & $18 \cdot 9$ & 2820 & $32 \cdot 7$ & 3089 & $40 \cdot 3$ & \\
\hline \multicolumn{8}{|l|}{ Education } \\
\hline Middle school or lower & 4391 & 43.9 & 3788 & $43 \cdot 1$ & 3363 & $37 \cdot 2$ & $<0.001$ \\
\hline High school & 3004 & $35 \cdot 8$ & 2803 & $32 \cdot 8$ & 2506 & $33 \cdot 1$ & \\
\hline College or higher & 1603 & $20 \cdot 3$ & 2089 & $24 \cdot 1$ & 2038 & $29 \cdot 7$ & \\
\hline
\end{tabular}

* Low income: monthly income $<$ minimum cost of living $\times 1.2$; middle income: minimum cost of living $\times 1.2 \leq$ monthly income $<$ minimum cost of living $\times 2.5$; high income: monthly income $\geqq$ minimum cost of living $\times 2.5$. 


\section{Change in vegetable consumption}

The results of the analyses of daily intake and the consumption ratio of vegetables are summarised in Table 2. There was no significant difference in total food intake among survey periods: $1267.5,1282.8$ and $1280.0 \mathrm{~g}$ in 1998,2001 and 2005 , respectively. In general, daily vegetable consumption in 1998, 2001 and 2005 was $293.5,305.7$ and $335.9 \mathrm{~g}$, indicating more than $30 \mathrm{~g}$ increase in 2005 compared with that in 1998 and 2001 in the study subjects, when male and female subjects were combined $(P<0.05$; Table 2$)$. Also, daily vegetable consumption by men and women separately increased in 2005 compared with that in 1998 and $2001(P<0.05)$. When taking into account daily vegetable consumption ratio (vegetable intake divided by total food intake), daily vegetable consumption by men and women separately increased gradually with a significant difference: $24.0 \%$ in $1998,24.8 \%$ in 2001 and $27.2 \%$ in 2005 $(P<0 \cdot 05$, data not shown) in the study subjects overall.

Vegetable consumption during lunch, in particular, increased significantly between the survey years $(P<0.05$; Table 2). Especially, vegetable consumption increased by $22.3 \mathrm{~g}$ in overall study subjects between 1998 and 2005. The intake for male subjects increased by $23.2 \mathrm{~g}$ and by $20.7 \mathrm{~g}$ in female subjects between the two study periods. Vegetable consumption ratio during breakfast decreased from $28.4 \%$ in 1998 to $24.9 \%$ in 2001 and $24.3 \%$ in $2005(P<0.05)$. This decreasing change was observed in both the male and female subjects. While vegetable consumption ratio during breakfast decreased, it increased during lunch from $34.3 \%$ in 1998 to $37.3 \%$ in 2001 and 2005, with similarity for both the male and female subjects $(P<0 \cdot 05)$. Vegetable consumption ratio during snack time also increased in terms of both daily intake and consumption ratio with time $(P<0.05)$.

Vegetable consumption and consumption ratios were analysed for each place of dining. Most meals were consumed in the home for all the survey years. Vegetable consumption at both restaurants and cafeterias increased significantly in 2001 and 2005 compared with that in 1998 in overall study subjects, and in the male and female subjects separately (Table 2). Specifically, the vegetable consumption ratio at cafeterias and restaurants increased from $14.0 \%$ and $11.5 \%$, respectively in 1998 to $18.1 \%$ and $17.4 \%$, respectively in 2005 , with similarity of increase in the male and female subjects, while the consumption of vegetables at home decreased from $66.5 \%$ in 1998 to $56.2 \%$ in 2005 in the male subjects and from $77.2 \%$ in 1998 to $66.7 \%$ in 2005 in the female subjects $(P<0 \cdot 05)$.

The results of vegetable consumption and consumption ratio analysed by the age group are summarised in Tables 3 and 4 . There is no significant difference for total food intake in all the age groups and between survey periods, except those aged 50-64 years who consumed a significantly higher intake $(1276.7 \mathrm{~g})$ in 2001 in comparison with that $(1184.9 \mathrm{~g})$ in 1998. In all the age groups, vegetable consumption increased by 33.7-53.2 g between 1998 and 2005. In general, the vegetable consumption ratio in all the age groups increased in 2005 compared with that in $1998(P<0 \cdot 05$, data not shown).

Analysis of changes in vegetable consumption by meal type revealed that from the ages of 7 to 12 years, vegetable consumption increased during both breakfast and lunch between 1998 and 2005, but for those aged 19-49 years, vegetable consumption decreased during breakfast $(P<0 \cdot 05$, Tables 3 and 4). Vegetable consumption during lunch increased for all the age groups and increased during dinner for all the age groups except those aged 7-15 years and $19-29$ years $(P<0 \cdot 05)$. In the $7-12$-year group, the ratio of vegetable consumption during dinner decreased from $34.0 \%$ in 1998 to $30.1 \%$ in $2005(P<0 \cdot 05)$. Analysis of vegetable consumption ratios for different meal types indicated decreased consumption during breakfast by the subjects over 13 years of age, though the consumption ratio during snack time increased for those over 30 years of age $(P<0.05)$. There was no significant difference between consumption during both lunch and dinner by the subjects over 50 years of age.

Analysis of vegetable consumption for different places of dining showed that vegetable consumption for those aged 7-12 years increased in cafeterias, restaurants and other places (Table 3). For those aged 7-15 and 19-49 years, vegetable consumption at home decreased, and for all ages consumption at both cafeterias and restaurants increased. Analysis of vegetable consumption ratio showed increased consumption in cafeterias, restaurants and other places and decreased consumption at home by the subjects aged 16-64 years with time $(P<0.05)$. For those aged over 65 years, there was a significant increase at restaurants and other places between 1998 and 2005 (Table 4).

\section{Discussion}

We analysed vegetable consumption in the Korean population between 1998 and 2005 and found that vegetable consumption increased. For the most part, the observed difference was similar in pattern between both the male and female subjects. Vegetable consumption increased more during lunch and dinner than during breakfast; the consumption ratio was found to have increased when the meal was consumed at a cafeteria or a restaurant. These changes were generally similar in both sex groups and between age groups, though some slight differences were observed.

A study on changes in food consumption in Portugal between 1987 and 1999 reported increasing trends in vegetable consumption by both men and women ${ }^{(11)}$. A study on trends in vegetable intake conducted on Swedish participants aged 70 years and above for a period of 30 years $(1971-2000)^{(18)}$ revealed that daily vegetable intake for men and women increased over the years $(92 \mathrm{~g} / \mathrm{d}$ for men; $84 \mathrm{~g} / \mathrm{d}$ for women). Another Swedish study, monitoring of trends and determinants in CVD, conducted in northern Sweden ${ }^{(19)}$, showed that between 1986 and 1999 Swedish adults increased the number of vegetable servings consumed and women increased their consumption by six servings per month in 1999 up from 29.1 servings in 1986. A study on food consumption in seven Mediterranean countries during the period of 1961-2001 also reported increased vegetable consumption between 30 and $174 \%$ in Algeria, Morocco, Turkey, Italy and Greece. This present study has similar results as previous studies: total vegetable consumption and vegetable consumption ratios increased from $24.0 \%$ in 1998 to $27.2 \%$ in 2005 . A notable thing in Korea is that Koreans 
Table 2. Comparison of vegetable consumption amount and intake ratio in 1998, 2001 and 2005 according to meal format by sex (Mean values with their standard errors)

\begin{tabular}{|c|c|c|c|c|c|c|c|c|c|c|c|c|c|c|c|c|c|c|}
\hline \multirow[t]{3}{*}{ Year... } & \multicolumn{6}{|c|}{ Total } & \multicolumn{6}{|c|}{ Male } & \multicolumn{6}{|c|}{ Female } \\
\hline & \multicolumn{2}{|c|}{1998} & \multicolumn{2}{|c|}{2001} & \multicolumn{2}{|c|}{2005} & \multicolumn{2}{|c|}{1998} & \multicolumn{2}{|c|}{2001} & \multicolumn{2}{|c|}{2005} & \multicolumn{2}{|c|}{1998} & \multicolumn{2}{|c|}{2001} & \multicolumn{2}{|c|}{2005} \\
\hline & Mean & SEM & Mean & SEM & Mean & SEM & Mean & SEM & Mean & SEM & Mean & SEM & Mean & SEM & Mean & SEM & Mean & SEM \\
\hline $\begin{array}{l}\text { Total food consump- } \\
\text { tion }(\mathrm{g} / \mathrm{d})\end{array}$ & $1267 \cdot 5$ & $14 \cdot 3$ & $1282 \cdot 8$ & 12.9 & $1280 \cdot 0$ & $13 \cdot 3$ & $1389 \cdot 8$ & $17 \cdot 1$ & $1380 \cdot 6$ & $15 \cdot 4$ & $1422 \cdot 4$ & $18 \cdot 0$ & $1154 \cdot 8$ & $14 \cdot 0$ & $1197 \cdot 2$ & $13 \cdot 7$ & $1139 \cdot 1$ & $12 \cdot 1$ \\
\hline $\begin{array}{l}\text { Total vegetable } \\
\text { consumption }(\mathrm{g} / \mathrm{d})\end{array}$ & $293 \cdot 5^{\mathrm{a}}$ & 0.4 & $305 \cdot 7^{\mathrm{a}}$ & 3.7 & $335 \cdot 9^{b}$ & 3.7 & $326 \cdot 8^{\mathrm{a}}$ & 4.9 & $335.7^{\mathrm{a}}$ & 4.4 & $368.9^{b}$ & $5 \cdot 0$ & $262 \cdot 4^{\mathrm{a}}$ & $4 \cdot 1$ & $279 \cdot 5^{\mathrm{b}}$ & 3.9 & $303 \cdot 3^{\mathrm{c}}$ & 3.8 \\
\hline \multicolumn{19}{|l|}{ Meal types } \\
\hline Breakfast & $84 \cdot 7$ & 1.6 & 79.6 & 1.6 & $83 \cdot 2$ & 1.6 & $91 \cdot 3^{\mathrm{a}}$ & 1.9 & $82.6^{\mathrm{b}}$ & 1.9 & $87 \cdot 1^{a, b}$ & $2 \cdot 0$ & 78.6 & 1.7 & $77 \cdot 0$ & 1.8 & 79.5 & $1 \cdot 7$ \\
\hline Lunch & $97 \cdot 5^{\mathrm{a}}$ & 1.5 & $110 \cdot 5^{b}$ & 1.5 & $119 \cdot 8^{c}$ & 1.5 & $109 \cdot 8^{\mathrm{a}}$ & 1.8 & $124 \cdot 3^{\mathrm{b}}$ & 1.9 & $133.0^{\mathrm{c}}$ & $2 \cdot 0$ & $86 \cdot 1^{\mathrm{a}}$ & $1 \cdot 7$ & $98 \cdot 4^{\mathrm{b}}$ & 1.6 & $106 \cdot 8^{c}$ & 1.8 \\
\hline Dinner & $102 \cdot 5^{\mathrm{a}}$ & 1.8 & $107 \cdot 9^{a}$ & 1.7 & $114 \cdot 0^{\mathrm{b}}$ & 1.8 & $115 \cdot 0^{\mathrm{a}}$ & $2 \cdot 3$ & $119 \cdot 8^{a}$ & $2 \cdot 0$ & $129 \cdot 4^{\mathrm{b}}$ & $2 \cdot 4$ & $91 \cdot 0^{\mathrm{a}}$ & 1.8 & $97 \cdot 4^{\mathrm{a}}$ & 1.9 & $98 \cdot 7^{b}$ & 1.8 \\
\hline Snack & 8.8 & 0.6 & $7 \cdot 7^{\mathrm{a}}$ & 0.5 & $18.9^{\mathrm{b}}$ & $1 \cdot 2$ & $10 \cdot 7^{a}$ & 0.8 & $8.9^{\mathrm{a}}$ & 0.7 & $19 \cdot 5^{\mathrm{b}}$ & 1.8 & $7.0^{\mathrm{a}}$ & 0.7 & $6 \cdot 7^{\mathrm{a}}$ & 0.6 & $18 \cdot 3^{\mathrm{b}}$ & 1.2 \\
\hline \multicolumn{19}{|l|}{ Ratio (\%)* } \\
\hline Breakfast & $28 \cdot 4^{\mathrm{a}}$ & 0.4 & $24 \cdot 9^{b}$ & 0.3 & $24 \cdot 3^{\mathrm{b}}$ & 0.3 & $27 \cdot 3^{\mathrm{a}}$ & 0.4 & $23 \cdot 4^{\mathrm{b}}$ & 0.4 & $22 \cdot 9^{\mathrm{b}}$ & 0.4 & $29 \cdot 4^{\mathrm{a}}$ & 0.4 & $26 \cdot 3^{\mathrm{b}}$ & 0.4 & $25 \cdot 6^{\mathrm{b}}$ & 0.4 \\
\hline Lunch & $34 \cdot 6^{a, b}$ & 0.4 & $35 \cdot 5^{\mathrm{a}}$ & 0.3 & $34 \cdot 2^{\mathrm{b}}$ & 0.4 & 34.8 & 0.4 & 35.6 & 0.4 & 35.3 & 0.4 & $34 \cdot 5^{a, b}$ & 0.5 & $35.4^{\mathrm{a}}$ & 0.4 & $33.2^{\mathrm{b}}$ & 0.5 \\
\hline Dinner & $34 \cdot 3^{a}$ & 0.3 & $37 \cdot 3^{b}$ & 0.4 & $37 \cdot 3^{\mathrm{b}}$ & 0.3 & $34.9^{\mathrm{a}}$ & 0.4 & $38.7^{\mathrm{b}}$ & 0.5 & $37.9^{\mathrm{b}}$ & 0.5 & $33 \cdot 7^{\mathrm{a}}$ & 0.5 & $36.0^{\mathrm{b}}$ & 0.4 & $36.7^{b}$ & 0.5 \\
\hline Snack & $2 \cdot 7^{\mathrm{a}}$ & 0.2 & $2 \cdot 4^{\mathrm{a}}$ & 0.2 & $4 \cdot 2^{\mathrm{b}}$ & 0.2 & $2 \cdot 9^{\mathrm{a}}$ & 0.2 & $2 \cdot 4^{a}$ & 0.2 & $4 \cdot 0^{\mathrm{b}}$ & 0.3 & $2 \cdot 5^{\mathrm{a}}$ & 0.2 & $2 \cdot 4^{a}$ & 0.2 & $4 \cdot 4^{\mathrm{b}}$ & 0.3 \\
\hline \multicolumn{19}{|l|}{ Dining locations } \\
\hline \multicolumn{19}{|l|}{ Amount $(\mathrm{g} / \mathrm{d})$} \\
\hline Home & $211 \cdot 9^{a}$ & $3 \cdot 4$ & $199 \cdot 3^{b}$ & 3.9 & $204 \cdot 5^{a, b}$ & 3.6 & $215 \cdot 9^{a}$ & $4 \cdot 1$ & $195 \cdot 0^{\mathrm{b}}$ & $4 \cdot 2$ & $202 \cdot 1^{a, b}$ & 4.7 & $208 \cdot 2$ & 3.9 & 203.0 & $4 \cdot 2$ & $206 \cdot 9$ & 3.9 \\
\hline Cafeteria & $37 \cdot 0$ & 1.5 & $46 \cdot 1^{\mathrm{b}}$ & 1.8 & $59 \cdot 1^{\mathrm{C}}$ & $2 \cdot 4$ & $48 \cdot 4^{\mathrm{a}}$ & $2 \cdot 2$ & $62 \cdot 7^{\mathrm{b}}$ & $2 \cdot 8$ & $76 \cdot 1^{\mathrm{C}}$ & 3.3 & $26 \cdot 5^{\mathrm{a}}$ & 1.3 & $31.5^{\mathrm{b}}$ & 1.4 & $42 \cdot 3^{\mathrm{C}}$ & $2 \cdot 2$ \\
\hline Restaurant & $37 \cdot 0^{\mathrm{a}}$ & 1.6 & $55 \cdot 3^{\mathrm{b}}$ & 1.9 & $62 \cdot 4^{\mathrm{b}}$ & $2 \cdot 2$ & $52 \cdot 7^{\mathrm{a}}$ & 2.6 & $72 \cdot 7^{\mathrm{b}}$ & $2 \cdot 6$ & $79 \cdot 7^{\mathrm{b}}$ & 3.3 & $22 \cdot 6^{\mathrm{a}}$ & 1.3 & $40 \cdot 1^{\mathrm{b}}$ & 1.9 & $45 \cdot 2^{\mathrm{b}}$ & 2.0 \\
\hline Other place & 7.5 & 0.9 & $5 \cdot 0^{\mathrm{b}}$ & 0.5 & $9 \cdot 9^{a}$ & 0.9 & $9 \cdot 8^{\mathrm{a}}$ & 1.3 & $5 \cdot 3^{b}$ & 0.7 & $11 \cdot 0^{\mathrm{a}}$ & 1.4 & $5 \cdot 5^{\mathrm{a}}$ & 0.6 & $4.8^{\mathrm{a}}$ & 0.6 & $8.9^{b}$ & 0.8 \\
\hline \multicolumn{19}{|l|}{ Ratio $(\%)^{\star}$} \\
\hline Home & $72 \cdot 1^{\mathrm{a}}$ & 0.6 & $65 \cdot 3^{\mathrm{b}}$ & 0.8 & $61.5^{\mathrm{c}}$ & 0.8 & $66.5^{\mathrm{a}}$ & 0.8 & $58.9^{b}$ & 0.9 & $56 \cdot 2^{\mathrm{b}}$ & 0.9 & $77 \cdot 2^{\mathrm{a}}$ & 0.7 & $71.0^{\mathrm{b}}$ & 0.8 & $66 \cdot 7^{\mathrm{C}}$ & 0.8 \\
\hline Cafeteria & $14 \cdot 0^{\mathrm{a}}$ & 0.5 & $16 \cdot 4^{\mathrm{b}}$ & 0.6 & $18 \cdot 1^{\mathrm{b}}$ & 0.7 & $16 \cdot 2^{\mathrm{a}}$ & 0.6 & $20 \cdot 0^{\mathrm{b}}$ & 0.7 & $20 \cdot 8^{\mathrm{b}}$ & 0.8 & $12 \cdot 0^{\mathrm{a}}$ & 0.5 & $13 \cdot 3^{\mathrm{a}}$ & 0.6 & $15 \cdot 5^{\mathrm{b}}$ & 0.7 \\
\hline Restaurant & $11.5^{\mathrm{a}}$ & 0.4 & $16 \cdot 6^{\mathrm{b}}$ & 0.5 & $17.4^{\mathrm{b}}$ & 0.6 & $14 \cdot 6^{\mathrm{a}}$ & 0.6 & $19 \cdot 6^{\mathrm{b}}$ & 0.7 & $20 \cdot 1^{\mathrm{b}}$ & 0.7 & $8 \cdot 6^{\mathrm{a}}$ & 0.4 & $13.9^{\mathrm{b}}$ & 0.6 & $14 \cdot 7^{\mathrm{b}}$ & 0.6 \\
\hline Other place & $2 \cdot 4^{\mathrm{a}}$ & 0.2 & $1.7^{b}$ & 0.2 & $3.0^{\mathrm{a}}$ & 0.2 & $2 \cdot 7^{\mathrm{a}}$ & 0.3 & $1.5^{\mathrm{b}}$ & 0.2 & $2 \cdot 9^{\mathrm{a}}$ & 0.3 & $2 \cdot 2^{\mathrm{a}}$ & 0.2 & $1.8^{\mathrm{a}}$ & 0.2 & $3.0^{\mathrm{b}}$ & 0.3 \\
\hline
\end{tabular}

a,b,c Mean values with unlike superscript letters within the same row are significantly different between years at $\alpha=0.05$ by Bonferroni's multiple $t$ test.

*Vegetable intake consumed during each meal or at each place of dining divided by total vegetable intake per $\mathrm{d}$. 
Table 3. Comparison of vegetable consumption amount and intake ratio in 1998, 2001 and 2005 according to meal format by age (7-29 years) (Mean values with their standard errors)

\begin{tabular}{|c|c|c|c|c|c|c|c|c|c|c|c|c|c|c|c|c|c|c|c|c|c|c|c|c|}
\hline \multirow{3}{*}{$\begin{array}{l}\text { Age (years). . } \\
\text { Year... }\end{array}$} & \multicolumn{6}{|c|}{$7-12$} & \multicolumn{6}{|c|}{$13-15$} & \multicolumn{6}{|c|}{$16-18$} & \multicolumn{6}{|c|}{$19-29$} \\
\hline & \multicolumn{2}{|l|}{1998} & \multicolumn{2}{|c|}{2001} & \multicolumn{2}{|c|}{2005} & \multicolumn{2}{|c|}{1998} & \multicolumn{2}{|l|}{2001} & \multicolumn{2}{|l|}{2005} & \multicolumn{2}{|c|}{1998} & \multicolumn{2}{|l|}{2001} & \multicolumn{2}{|c|}{2005} & \multicolumn{2}{|c|}{1998} & \multicolumn{2}{|l|}{2001} & \multicolumn{2}{|l|}{2005} \\
\hline & Mean & SEM & Mean & SEM & Mean & SEM & Mean & SEM & Mean & SEM & Mean & SEM & Mean & SEM & Mean & SEM & Mean & SEM & Mean & SEM & Mean & SEM & Mean & SEM \\
\hline $\begin{array}{l}\text { Total food } \\
\text { consumption } \\
\text { (g/d) }\end{array}$ & 1167.5 & $24 \cdot 1$ & $1143 \cdot 0$ & 19.5 & $1148 \cdot 3$ & $18 \cdot 2$ & 1263.6 & 33.7 & 1218.8 & $25 \cdot 2$ & 1243.7 & 29.0 & $1210 \cdot 8$ & 31.0 & $1226 \cdot 8$ & 31.7 & 1298.5 & 34.4 & 1326.2 & 22.5 & $13320 \cdot 0$ & $25 \cdot 1$ & 1399.5 & 30. \\
\hline $\begin{array}{l}\text { Total vegetable } \\
\text { consumption } \\
(\mathrm{g} / \mathrm{d})\end{array}$ & $174 \cdot 6^{a}$ & 6.1 & $173 \cdot 4^{a}$ & 4.8 & $212 \cdot 0^{\mathrm{b}}$ & $6 \cdot 3$ & $218 \cdot 5^{a}$ & 8.3 & $215 \cdot 7^{\mathrm{a}}$ & 7.8 & $264 \cdot 3^{\mathrm{b}}$ & 9.7 & $216 \cdot 5^{\mathrm{a}}$ & 6.9 & $236 \cdot 2^{a, b}$ & 9.1 & $262.5^{\mathrm{b}}$ & $13 \cdot 2$ & $285 \cdot 6^{a}$ & 6.2 & $289 \cdot 0^{a}$ & 5.9 & $328 \cdot 3^{b}$ & 8.4 \\
\hline \multicolumn{25}{|l|}{ Meal types } \\
\hline \multicolumn{25}{|l|}{ Amount $(\mathrm{g} / \mathrm{d})$} \\
\hline Lunch & $63 \cdot 4^{\mathrm{a}}$ & $\begin{array}{l}2.4 \\
2.4\end{array}$ & $66.9^{\mathrm{a}}$ & 2.4 & $86.9^{\mathrm{b}}$ & $\begin{array}{l}2.0 \\
2.9\end{array}$ & $65 \cdot 7^{\mathrm{a}}$ & 4.0 & $84.1^{\mathrm{b}}$ & $\begin{array}{l}3.0 \\
3.8\end{array}$ & $101 \cdot 6^{\mathrm{c}}$ & 4.6 & $70 \cdot 9^{\mathrm{a}}$ & $\begin{array}{l}3.0 \\
3.3\end{array}$ & $93 \cdot 1^{\mathrm{b}}$ & 4.6 & $99.8^{\mathrm{b}}$ & $\begin{array}{l}5.0 \\
5.4\end{array}$ & $100 \cdot 7^{\mathrm{a}}$ & $\begin{array}{l}2.0 \\
2.8\end{array}$ & $115.7^{\mathrm{b}}$ & 3.0 & $131.8^{\mathrm{c}}$ & 4.4 \\
\hline Dinner & $62 \cdot 1$ & 3.3 & 62.4 & 2.5 & 62.9 & $3 \cdot 1$ & 82.7 & 4.4 & 76.4 & 3.9 & 91.5 & 5.8 & $72 \cdot 8^{\mathrm{a}}$ & 3.3 & $84 \cdot 6^{a, b}$ & 4.4 & $89.4^{\mathrm{b}}$ & 5.1 & 106.5 & $3 \cdot 2$ & $109 \cdot 4$ & 3.4 & $118 \cdot 3$ & 4.1 \\
\hline Snack & $6 \cdot 8^{\mathrm{a}}$ & 1.2 & $5 \cdot 6^{a}$ & 0.8 & $13 \cdot 2^{\mathrm{b}}$ & 1.8 & 9.1 & 1.9 & $7 \cdot 2$ & 1.4 & 14.0 & 3.4 & $12 \cdot 2$ & 1.8 & 9.3 & 2.4 & 19.6 & 5.0 & $10 \cdot 3^{\mathrm{a}}$ & 1.1 & $9 \cdot 6^{a}$ & 1.3 & $20.5^{\mathrm{b}}$ & 3.5 \\
\hline \multicolumn{25}{|l|}{ Ratio $(\%)^{*}$} \\
\hline Breakfast & 22.8 & 0.9 & 20.8 & 0.7 & 22.5 & 0.9 & $27 \cdot 1^{\mathrm{a}}$ & 1.2 & $20.5^{\mathrm{b}}$ & $1 \cdot 2$ & $21 \cdot 7^{\mathrm{b}}$ & 1.3 & $27 \cdot 5^{\mathrm{a}}$ & 1.3 & $19.5^{\mathrm{b}}$ & 1.6 & $18.7^{\mathrm{b}}$ & 1.6 & $22 \cdot 8^{\mathrm{a}}$ & 0.7 & $18.1^{\mathrm{b}}$ & 0.7 & $16 \cdot 6^{\mathrm{b}}$ & $0 . c$ \\
\hline Lunch & 39.8 & 1.2 & 41.6 & $1 \cdot 1$ & $43 \cdot 1$ & 1.2 & $32 \cdot 2^{\mathrm{a}}$ & 1.4 & $41 \cdot 6^{\mathrm{b}}$ & 1.4 & $41 \cdot 2^{\mathrm{b}}$ & 1.5 & $32 \cdot 4^{\mathrm{a}}$ & 1.3 & $40.5^{b}$ & 1.6 & $40 \cdot 6^{b}$ & b. 2.2 & $36.5^{\mathrm{a}}$ & 0.8 & $40 \cdot 8^{\mathrm{b}}$ & 0.9 & $41.7^{\mathrm{b}}$ & $1 . \mathrm{C}$ \\
\hline Dinner & $34.0^{\mathrm{a}}$ & 1.0 & $34.8^{\mathrm{a}}$ & 1.0 & $30.1^{\mathrm{b}}$ & 0.9 & 36.8 & 1.5 & 34.6 & $1 \cdot 2$ & 33.6 & 1.3 & 35.1 & 1.2 & 36.7 & 1.6 & 35.4 & 1.6 & 37.1 & 0.9 & 38.0 & 0.8 & 37.1 & 0.5 \\
\hline Snack & $3 \cdot 3^{\mathrm{a}, \mathrm{b}}$ & 0.4 & $2 \cdot 9^{a}$ & 0.4 & $4 \cdot 3^{\mathrm{b}}$ & 0.5 & 3.9 & 0.7 & 3.3 & 0.5 & 3.5 & 0.7 & 5.0 & 0.7 & 3.3 & 0.7 & $5 \cdot 3$ & 1.0 & 3.6 & 0.3 & 3.1 & 0.5 & 4.5 & 0.5 \\
\hline \multirow{2}{*}{\multicolumn{25}{|c|}{ Dining locations }} \\
\hline & & & & & & & & & & & & & & & & & & & & & & & & \\
\hline Home & $119 \cdot 5^{\mathrm{a}, \mathrm{b}}$ & 5.5 & $110 \cdot 6^{a}$ & 4.2 & $113 \cdot 0^{\mathrm{b}}$ & $5 \cdot 8$ & $165 \cdot 8^{a}$ & 8.1 & $139 \cdot 3^{\mathrm{b}}$ & 6.7 & $147 \cdot 2^{a, b}$ & 8.9 & 149.5 & 6.9 & 139.3 & 8.9 & 128.0 & 10.5 & $182 \cdot 1^{a}$ & 6.0 & $148 \cdot 4^{b}$ & $6 \cdot 1$ & $157.5^{b}$ & $7 \cdot 0$ \\
\hline Cafeteria & $47 \cdot 6^{\mathrm{a}}$ & 2.6 & $49.8^{\mathrm{a}}$ & $2 \cdot 7$ & $60.8^{\mathrm{b}}$ & 4.1 & $41 \cdot 7^{\mathrm{a}}$ & 3.8 & $60.4^{\mathrm{b}}$ & 4.4 & $84 \cdot 4^{\mathrm{C}}$ & 6.9 & $10 \cdot 6^{\mathrm{b}}$ & 4.7 & $52.5^{\mathrm{a}}$ & 5.5 & $88 \cdot 1^{b}$ & 8.2 & $41 \cdot 4^{\mathrm{a}}$ & 3.2 & $54 \cdot 8^{\mathrm{b}}$ & 3.8 & $73 \cdot 3^{\mathrm{c}}$ & 5.5 \\
\hline Restaurant & $5.0^{\mathrm{a}}$ & $1 \cdot 1$ & $9 \cdot 9^{\mathrm{b}}$ & 1.4 & $11 \cdot 4^{\mathrm{b}}$ & 1.9 & $9.5^{\mathrm{a}}$ & $2 \cdot 2$ & $15 \cdot 0^{\mathrm{a}}$ & $2 \cdot 1$ & $22 \cdot 0^{\mathrm{b}}$ & 4.6 & $19 \cdot 2^{\mathrm{a}}$ & 2.4 & $42 \cdot 7^{b}$ & $5 \cdot 1$ & $37.5^{\mathrm{b}}$ & 4.5 & $52 \cdot 8^{\mathrm{a}}$ & 3.5 & $79 \cdot 9^{b}$ & 4.7 & $88 \cdot 3^{\mathrm{b}}$ & 5.7 \\
\hline Other place & $2 \cdot 4^{\mathrm{a}}$ & 0.8 & $3.1^{\mathrm{a}}$ & 1.0 & $6 \cdot 7^{b}$ & 1.2 & $1.5^{\mathrm{a}}$ & 0.8 & $1 \cdot 1^{\mathrm{a}}$ & 0.4 & $10 \cdot 6^{\mathrm{b}}$ & 4.7 & $3 \cdot 6^{\mathrm{a}, \mathrm{b}}$ & 1.1 & $1 \cdot 6^{\mathrm{a}}$ & 0.8 & $8.9^{b}$ & 2.8 & 9.3 & 1.3 & $5 \cdot 9$ & $1 \cdot 1$ & 9.2 & 3.1 \\
\hline \multicolumn{25}{|l|}{ Ratio $(\%)^{*}$} \\
\hline Home & 65.4 & 1.6 & $61 \cdot 1$ & 1.4 & 60.4 & 1.7 & $72 \cdot 4^{\mathrm{a}}$ & 1.7 & $62 \cdot 3^{\mathrm{b}}$ & 2.0 & $57.0^{\mathrm{b}}$ & 2.5 & $69.7^{\mathrm{a}}$ & $1 \cdot 7$ & $58 \cdot 8^{\mathrm{b}}$ & $2 \cdot 7$ & $48.5^{\mathrm{c}}$ & $=2.7$ & $62 \cdot 2^{\mathrm{a}}$ & 1.2 & $52 \cdot 4^{\mathrm{b}}$ & 1.4 & $50 \cdot 6^{\mathrm{b}}$ & 1.6 \\
\hline Cafeteria & 30.7 & 1.6 & $31 \cdot 1$ & 1.4 & 30.4 & 1.8 & $22 \cdot 4^{\mathrm{a}}$ & 1.7 & $30 \cdot 3^{\mathrm{b}}$ & 1.9 & $31 \cdot 1^{\mathrm{b}}$ & 2.2 & $19.7^{\mathrm{a}}$ & 1.5 & $23 \cdot 6^{\mathrm{a}}$ & $2 \cdot 2$ & $33.4^{\mathrm{b}}$ & $2 \cdot 6$ & $15 \cdot 0^{\mathrm{a}}$ & 1.0 & $19 \cdot 3^{\mathrm{b}}$ & $1 \cdot 2$ & $21 \cdot 6^{\mathrm{b}}$ & 1.5 \\
\hline Restaurant & $2 \cdot 6^{\mathrm{a}}$ & 0.5 & $6.1^{\mathrm{b}}$ & 0.7 & $5.7^{\mathrm{b}}$ & 0.9 & 4.7 & 0.9 & 6.9 & 1.0 & 8.2 & 1.3 & $8 \cdot 8^{\mathrm{a}}$ & $1 \cdot 1$ & $17 \cdot 0^{\mathrm{b}}$ & 1.9 & $15 \cdot 3^{\mathrm{b}}$ & 2.0 & $19 \cdot 2^{\mathrm{a}}$ & 1.0 & $26 \cdot 5^{\mathrm{b}}$ & 1.2 & $25 \cdot 6^{\mathrm{b}}$ & 1.4 \\
\hline Other place & $1.3^{\mathrm{a}}$ & 0.4 & $1.7^{\mathrm{a}}$ & 0.5 & $3.5^{\mathrm{b}}$ & 0.6 & 0.4 & 0.2 & 0.5 & 0.2 & 3.7 & 1.5 & $1 \cdot 7^{\mathrm{a}, \mathrm{b}}$ & 0.5 & $0.6^{\mathrm{a}}$ & 0.3 & $2 \cdot 8^{\mathrm{b}}$ & 0.8 & $3.5^{\mathrm{a}}$ & 0.4 & $1.9^{b}$ & 0.3 & $2 \cdot 3^{\mathrm{a}, \mathrm{b}}$ & 0.4 \\
\hline
\end{tabular}

${ }^{\mathrm{a}, \mathrm{b}, \mathrm{c}}$ Mean values with unlike superscript letters within the same row are significantly different between years at $\alpha=0.05$ by Bonferroni's multiple $t$ test.

* Vegetable intake consumed during each meal or at each place of dining divided by total vegetable intake per $d$. 
Table 4. Comparison of vegetable consumption amount and intake ratio in 1998, 2001 and 2005 according to meal format by age ( 30 years and over) (Mean values with their standard errors)

\begin{tabular}{|c|c|c|c|c|c|c|c|c|c|c|c|c|c|c|c|c|c|c|}
\hline \multirow{3}{*}{$\begin{array}{l}\text { Age (years)... } \\
\text { Year... }\end{array}$} & \multicolumn{6}{|c|}{$30-49$} & \multicolumn{6}{|c|}{$50-64$} & \multicolumn{6}{|c|}{$\geq 65$} \\
\hline & \multicolumn{2}{|c|}{1998} & \multicolumn{2}{|c|}{2001} & \multicolumn{2}{|c|}{2005} & \multicolumn{2}{|c|}{1998} & \multicolumn{2}{|c|}{2001} & \multicolumn{2}{|c|}{2005} & \multicolumn{2}{|c|}{1998} & \multicolumn{2}{|c|}{2001} & \multicolumn{2}{|c|}{2005} \\
\hline & Mean & SEM & Mean & SEM & Mean & SEM & Mean & SEM & Mean & SEM & Mean & SEM & Mean & SEM & Mean & SEM & Mean & SEM \\
\hline $\begin{array}{l}\text { Total food consump- } \\
\text { tion }(\mathrm{g} / \mathrm{d})\end{array}$ & $1377 \cdot 6$ & $19 \cdot 0$ & $1394 \cdot 9$ & $17 \cdot 7$ & $1365 \cdot 0$ & $19 \cdot 2$ & $1184.9^{a}$ & $21 \cdot 1$ & $1276 \cdot 7^{b}$ & $21 \cdot 8$ & $1227 \cdot 6^{a, b}$ & $19 \cdot 4$ & $964 \cdot 5$ & $23 \cdot 2$ & $1021 \cdot 0$ & $22 \cdot 8$ & $968 \cdot 4$ & $17 \cdot 8$ \\
\hline $\begin{array}{l}\text { Total vegetable } \\
\text { consumption }(\mathrm{g} / \mathrm{d})\end{array}$ & $347 \cdot 1^{a}$ & 5.9 & $356 \cdot 7^{\mathrm{a}}$ & $5 \cdot 2$ & $380 \cdot 8^{\mathrm{b}}$ & $5 \cdot 4$ & $320 \cdot 7^{a}$ & 6.9 & $353.9^{b}$ & $8 \cdot 7$ & $373 \cdot 9^{b}$ & $7 \cdot 8$ & $267 \cdot 9^{a}$ & $8 \cdot 3$ & $296 \cdot 6^{a, b}$ & 8.6 & $310 \cdot 2^{b}$ & 8.4 \\
\hline \multicolumn{19}{|l|}{ Meal types } \\
\hline \multicolumn{19}{|l|}{ Amount $(\mathrm{g} / \mathrm{d})$} \\
\hline Breakfast & $99 \cdot 2^{\mathrm{a}}$ & $2 \cdot 4$ & $91 \cdot 0^{\mathrm{b}}$ & $2 \cdot 4$ & $92 \cdot 3^{b}$ & $2 \cdot 3$ & $107 \cdot 4$ & $3 \cdot 2$ & $112 \cdot 6$ & 3.9 & $117 \cdot 2$ & 3.8 & 98.5 & $3 \cdot 7$ & 101.4 & $3 \cdot 7$ & $101 \cdot 2$ & 3.0 \\
\hline Lunch & $116 \cdot 1^{a}$ & $2 \cdot 2$ & $129 \cdot 8^{b}$ & $2 \cdot 2$ & $134.4^{\mathrm{b}}$ & 2.4 & $100 \cdot 8^{a}$ & $2 \cdot 9$ & $115 \cdot 7^{b}$ & $3 \cdot 2$ & $118 \cdot 6^{b}$ & $3 \cdot 1$ & $81 \cdot 3^{\mathrm{a}}$ & 3.3 & $94.5^{\mathrm{b}}$ & $3 \cdot 7$ & $94 \cdot 8^{b}$ & 2.9 \\
\hline Dinner & $121.9^{a}$ & $2 \cdot 8$ & $127 \cdot 4^{\mathrm{a}, \mathrm{b}}$ & $2 \cdot 3$ & $132.4^{b}$ & $2 \cdot 8$ & $106 \cdot 1^{a}$ & $3 \cdot 2$ & $117.9^{a, b}$ & $3 \cdot 8$ & $119 \cdot 3^{b}$ & $3 \cdot 2$ & $83 \cdot 6^{\mathrm{a}}$ & $3 \cdot 2$ & $96 \cdot 5^{\mathrm{b}}$ & $3 \cdot 2$ & $100 \cdot 7^{b}$ & 3.4 \\
\hline Snack & $9 \cdot 9^{a}$ & 1.2 & $8 \cdot 5^{\mathrm{a}}$ & 0.8 & $21 \cdot 6^{\mathrm{b}}$ & 1.7 & $6 \cdot 4^{a}$ & 1.0 & $7 \cdot 6^{\mathrm{a}}$ & 1.4 & $18.9^{b}$ & $2 \cdot 1$ & $4.5^{\mathrm{a}}$ & 0.9 & $4 \cdot 2^{\mathrm{b}}$ & $1 \cdot 1$ & $13 \cdot 5^{\mathrm{b}}$ & $2 \cdot 4$ \\
\hline \multicolumn{19}{|l|}{ Ratio $(\%)^{*}$} \\
\hline Breakfast & $28 \cdot 8^{\mathrm{a}}$ & 0.5 & $24.7^{\mathrm{b}}$ & 0.5 & $24 \cdot 0^{\mathrm{b}}$ & 0.5 & $33.6^{\mathrm{a}}$ & 0.7 & $31 \cdot 8^{\mathrm{a}, \mathrm{b}}$ & 0.7 & $31 \cdot 2^{\mathrm{b}}$ & 0.7 & $37.5^{\mathrm{a}}$ & 0.7 & $34.2^{b}$ & 0.7 & $33.2^{b}$ & 0.6 \\
\hline Lunch & $34.4^{\mathrm{a}}$ & 0.5 & $36.9^{\mathrm{b}}$ & 0.5 & $36 \cdot 3^{\mathrm{b}}$ & 0.5 & 31.5 & 0.6 & 33.0 & 0.6 & $32 \cdot 7$ & 0.6 & $30 \cdot 2$ & 0.7 & 31.2 & 0.9 & 31.0 & 0.7 \\
\hline Dinner & $34 \cdot 6^{\mathrm{a}}$ & 0.5 & $36 \cdot 1^{\mathrm{b}}$ & 0.4 & $35 \cdot 1^{\mathrm{a}, \mathrm{b}}$ & 0.5 & $33 \cdot 3$ & 0.6 & 33.4 & 0.7 & $32 \cdot 3$ & 0.7 & 30.9 & 0.7 & 33.4 & 0.8 & 32.9 & 0.6 \\
\hline Snack & $2 \cdot 3^{\mathrm{a}}$ & 0.2 & $2 \cdot 2^{\mathrm{a}}$ & 0.2 & $4 \cdot 6^{\mathrm{a}, \mathrm{b}}$ & 0.3 & $1.6^{\mathrm{a}}$ & 0.2 & $1.9^{\mathrm{a}}$ & 0.3 & $3.7^{b}$ & 0.4 & $1.4^{\mathrm{a}}$ & 0.3 & $1 \cdot 2^{\mathrm{a}}$ & 0.2 & $2 \cdot 8^{\mathrm{b}}$ & 0.4 \\
\hline \multicolumn{19}{|l|}{ Dining locations } \\
\hline \multicolumn{19}{|l|}{ Amount $(\mathrm{g} / \mathrm{d})$} \\
\hline Home & $241 \cdot 2^{\mathrm{a}}$ & $5 \cdot 1$ & $217 \cdot 0^{\mathrm{b}}$ & 5.0 & $217 \cdot 1^{\mathrm{b}}$ & 5.4 & $256 \cdot 6$ & $6 \cdot 4$ & $266 \cdot 4$ & 8.9 & $268 \cdot 3$ & 7.9 & $250 \cdot 2$ & $8 \cdot 1$ & 264.7 & 8.5 & $272 \cdot 3$ & $8 \cdot 1$ \\
\hline Cafeteria & $41.0^{\mathrm{a}}$ & $2 \cdot 2$ & $54 \cdot 3^{\mathrm{b}}$ & 2.9 & $65 \cdot 3^{c}$ & $3 \cdot 1$ & $26 \cdot 7^{\mathrm{a}}$ & $2 \cdot 7$ & $32 \cdot 4^{a, b}$ & 3.0 & $40 \cdot 6^{b}$ & 3.5 & $7 \cdot 1^{a}$ & $1 \cdot 7$ & $9 \cdot 3^{a, b}$ & 1.8 & $13 \cdot 8^{b}$ & $2 \cdot 2$ \\
\hline Restaurant & $54 \cdot 0^{\mathrm{a}}$ & $2 \cdot 8$ & $79.8^{b}$ & 2.9 & $86 \cdot 4^{\mathrm{b}}$ & $3 \cdot 4$ & $30 \cdot 4^{a}$ & 2.9 & $47 \cdot 6^{\mathrm{b}}$ & 3.7 & $53 \cdot 7^{\mathrm{b}}$ & 3.6 & $7 \cdot 8^{\mathrm{a}}$ & 1.5 & $18 \cdot 8^{\mathrm{b}}$ & 2.6 & $19 \cdot 5^{\mathrm{b}}$ & 2.5 \\
\hline Other place & $10 \cdot 9^{\mathrm{a}}$ & 1.7 & $5 \cdot 6^{\mathrm{b}}$ & 0.8 & $12 \cdot 0^{\mathrm{a}}$ & 1.4 & 6.9 & 1.5 & 7.4 & 1.3 & $11 \cdot 3$ & 1.6 & 2.9 & 0.7 & 3.7 & $1 \cdot 1$ & 4.6 & 1.0 \\
\hline \multicolumn{19}{|l|}{ Ratio $(\%)^{\star}$} \\
\hline Home & $70 \cdot 4^{a}$ & 0.8 & $61.9^{\mathrm{b}}$ & 0.9 & $57.8^{\mathrm{b}}$ & 0.9 & $81 \cdot 7^{\mathrm{a}}$ & 1.0 & $76 \cdot 4^{\mathrm{b}}$ & $1 \cdot 1$ & $71 \cdot 4^{\mathrm{C}}$ & $1 \cdot 1$ & $93.1^{\mathrm{a}}$ & 0.9 & $89 \cdot 6^{\mathrm{b}}$ & 1.0 & $88.0^{\mathrm{b}}$ & 1.0 \\
\hline Cafeteria & $12 \cdot 0^{\mathrm{a}}$ & 0.6 & $14 \cdot 8^{\mathrm{b}}$ & 0.7 & $16 \cdot 9^{\mathrm{b}}$ & 0.8 & $7 \cdot 6^{\mathrm{a}}$ & 0.7 & $8 \cdot 7^{\mathrm{a}, \mathrm{b}}$ & 0.6 & $10 \cdot 4^{\mathrm{b}}$ & 0.8 & 2.7 & 0.5 & 2.9 & 0.6 & $4 \cdot 0$ & 0.6 \\
\hline Restaurant & $14 \cdot 6^{\mathrm{a}}$ & 0.6 & $21 \cdot 6^{\mathrm{b}}$ & 0.7 & $22 \cdot 1^{\mathrm{b}}$ & 0.8 & $8 \cdot 7^{\mathrm{a}}$ & 0.7 & $12 \cdot 4^{\mathrm{b}}$ & 0.9 & $14 \cdot 8^{\mathrm{b}}$ & 0.9 & $2 \cdot 8^{a}$ & 0.5 & $6 \cdot 2^{b}$ & 0.8 & $6 \cdot 2^{\mathrm{b}}$ & 0.7 \\
\hline Other place & $3 \cdot 0^{\mathrm{a}}$ & 0.4 & $1.7^{\mathrm{b}}$ & 0.2 & $3 \cdot 2^{\mathrm{a}}$ & 0.4 & $2 \cdot 0^{\mathrm{a}}$ & 0.3 & $2 \cdot 5^{\mathrm{b}}$ & 0.4 & $3.4^{\mathrm{c}}$ & 0.5 & $1.4^{\mathrm{a}}$ & 0.3 & $1 \cdot 3^{b}$ & 0.3 & $1.8^{\mathrm{b}}$ & 0.4 \\
\hline
\end{tabular}

${ }^{a, b, c}$ Mean values with unlike superscript letters within the same row are significantly different between years at $\alpha=0.05$ by Bonferroni's multiple $t$ test.

* Vegetable intake consumed during each meal or at each place of dining divided by total vegetable intake per $\mathrm{d}$. 
consume a relatively high intake of fermented vegetables. For example, daily intakes of Kimchi in Korea were $131.8 \mathrm{~g}$ in $1998,136.3 \mathrm{~g}$ in 2001 and $129.1 \mathrm{~g}$ in 2005 , which are $40-45 \%$ of daily total vegetable intake consumed by Koreans (data not shown). However, the consumption ratio of Kimchi decreased in 2005 compared with that in 1998 and in 2001, while the consumption ratio of fresh vegetables had an increasing change with time.

There are some studies conducted in the USA, Spain and Australia ${ }^{(9,10,20,21)}$ that have revealed decreasing trends in vegetable consumption. Some researchers argue that increases in vegetable consumption are closely related to socioeconomic factors. Researchers analysing data from NHANES found that the vegetable consumption ratio differed between whites and blacks in the USA and found that the ratio increased as economic and educational levels became higher in both whites and blacks ${ }^{(22)}$. Researchers in Portugal have also found a link between increased vegetable consumption and levels of education when taking location and age into account $^{(11)}$. Similarly, in the present study we found that while vegetable consumption increased, so did the levels of education and economic status of subjects between 1998 and 2005, suggesting that socioeconomic status may be correlated with vegetable consumption behaviours. In fact, the international monetary fund crisis in December 1997 was a painful blow to Koreans that resulted in a major economic recession that affected the food intake situation. Therefore, the food consumption was low in 1998, and there has been economic improvement resulting in vegetable intake increase since then.

While in our study vegetable consumption in all the age groups is increasing, other studies have shown the opposite. For instance, a study in the USA on dietary habits of preschoolers using the National Food Consumption Survey for 1977-9 and the Continuing Survey of Food Intakes by Individuals data for 1989-91, 1994-6 and $1998^{(23)}$ reported increased vegetable servings in 1994-96 and 1998 compared with those in 1977-79. A similar study of children aged 9-10 years in Liverpool, $\mathrm{UK}^{(24)}$ also reported increased vegetable consumption ratios during the period from 2001 to 2005 (boys, 23.5\%; girls, 44.8\%). However, another study in the USA on trends in dietary patterns of fifth graders (approximately 10 years of age) in Bogalusa, USA from 1973 until $1994^{(25,26)}$, showed that vegetable consumption ratios decreased and average vegetable consumption decreased from $146.5 \mathrm{~g}$ in $1973-4$ to $134.1 \mathrm{~g}$ in $1993-4$, though there was not a statistically significant difference. Another study conducted in the same area on trends in dietary patterns in the subjects transitioning from childhood to adulthood also reported no significant changes in vegetable consumption patterns. A study on the vegetable and fruit consumption patterns of juveniles in Minnesota, USA between 1994 and 2004 found that the number of servings of vegetables (green/orange vegetables) consumed by early to middle adolescents decreased in both the male and female subjects, and the decrease in the number of servings of green/orange vegetables consumed was observed only in the male subjects in middle to late adolescence. A trend of decreasing numbers of vegetable servings in both men and women was reported in a study using data from the Behavioural Risk Factor Surveillance System in the $\mathrm{USA}^{(9)}$. But the same study also reported inconsistent trends of daily vegetable and fruit consumption ratios between different age groups. Consumption ratios increased in the male subjects between the ages of 18 and 24 years and decreased in the male subjects between the ages of 55 and 64 years. At the same time, the consumption ratios increased in the female subjects aged 18-34 years and decreased in those aged 65 years or older. One study ${ }^{(18,27)}$ reported an increasing trend of vegetable intake in subjects aged 60 years or older. The first studies to examine trends of vegetable intake have varied from region to region, for example, a trend of decreased vegetable intake in the USA $v$. a trend of increased vegetable intake in European countries. Discrepancies in trends also exist between the age groups. While a trend showed increased vegetable intake in adults aged 18-24 years in the USA, the trend was decreasing in other age groups.

In our study, we looked into the pattern of changes in vegetable consumption in the context of different meal types and places of dining. In general, vegetable consumption did not change for breakfast. But consumption did increase for lunch and dinner, which was similar in both men and women. In children aged 7-12 years, vegetable consumption increased for breakfast and lunch, while no change in vegetable intake for each mealtime was observed in the study of children in Bogalusa, USA ${ }^{(25)}$. Also, in adults aged 19-49 years, vegetable consumption during breakfast decreased while consumption increased during lunch. These finding indicate that vegetable consumption varies by meal type and age.

Changes in vegetable consumption patterns are thought to be influenced by an increasing trend of skipping breakfast and/or replacing it with fast alternatives while rushing in the morning. In fact, $38 \%$ of those between the ages of 20 and 29 years and $17 \cdot 2 \%$ of those between the ages of 30 and 49 years skip breakfast in the morning, according to the 2005 KNHANES report ${ }^{(28)}$. Fewer children (aged 7-12 years) and older adults ( $>50$ years of age) were found to skip breakfast. Analysis of vegetable consumption in different places of dining showed increasing trends of vegetable consumption and consumption ratio at cafeterias and restaurants. Generally, meals eaten at home had the highest vegetable consumption ratio compared with other places of dining, though this trend changed in 2005, and vegetable consumption during meals at home decreased, while vegetable consumption at cafeterias and restaurants increased. The study of children of about 10 years of age in Bogalusa, USA ${ }^{(25)}$ reported that it did show that vegetable consumption during meals eaten at school or at restaurants increased. Also in the Bogalusa study, there were no substantial changes observed in vegetable consumption during meals eaten at home, there was an increasing trend of vegetable consumption during meals eaten outside of the home.

The overall results of our analysis indicate that vegetable consumption by Koreans has steadily increased. These differences are considered to have been influenced by changing factors such as improved socioeconomic status, advancement of the food industry and other factors related to dietary habit. However, there have been few studies on comparison in vegetable consumption behaviours related to these factors, particularly with factors like socioeconomic status and changes in standards of living. Therefore, further studies on changes in vegetable consumption for people of different socioeconomic backgrounds will be necessary in the future. 


\section{Acknowledgements}

The present study was supported by the National Cancer Center (0731060-1 and 0910221-1).

J.-S. L. performed the statistical analysis and participated in drafting the manuscript. J. K. conceived of the study, participated in its design and coordination and revised the manuscript critically for important intellectual content. Both the authors read and approved the final manuscript.

The authors state that there are no conflicts of interest.

\section{References}

1. Bes-Rastrollo M, Martinez-Gonzalez MA, Sanchez-Villegas A, et al. (2006) Association of fiber intake and fruit/vegetable consumption with weight gain in a Mediterranean population. Nutrition 22, 504-511.

2. Nakamura K, Nagata C, Oba S, et al. (2008) Fruit and vegetable intake and mortality from cardiovascular disease are inversely associated in Japanese women but not in men. $J$ Nutr 138, 1129-1134.

3. Hung HC, Joshipura KJ, Jiang R, et al. (2004) Fruit and vegetable intake and risk of major chronic disease. J Natl Cancer Inst 96, 1577-1584.

4. Steffen LM, Jacobs DR Jr, Stevens J, et al. (2003) Associations of whole-grain, refined-grain, and fruit and vegetable consumption with risks of all-cause mortality and incident coronary artery disease and ischemic stroke: The Atherosclerosis Risk in Communities (ARIC) Study. Am J Clin Nutr 78, 383-390.

5. Lunet N, Valbuena C, Vieira AL, et al. (2007) Fruit and vegetable consumption and gastric cancer by location and histological type: case-control and meta-analysis. Eur $J$ Cancer Prev 16, 312-327.

6. Maynard M, Gunnell D, Emmett P, et al. (2003) Fruit, vegetables, and antioxidants in childhood and risk of adult cancer: the Boyd Orr cohort. J Epidemiol Commun Health 57, 218-225.

7. Steffen LM, Kroenke CH, Yu X, et al. (2005) Associations of plant food, dairy product, and meat intakes with 15 -y incidence of elevated blood pressure in young black and white adults: The Coronary Artery Risk Development in Young Adults (CARDIA) Study. Am J Clin Nutr 82, 1169-1177, quiz 1363-1164.

8. Ford ES \& Mokdad AH (2001) Fruit and vegetable consumption and diabetes mellitus incidence among U.S. adults. Prev Med 32, 33-39.

9. Blanck HM, Gillespie C, Kimmons JE, et al. (2008) Trends in fruit and vegetable consumption among U.S. men and women, 1994-2005. Prev Chronic Dis 5, A35.

10. Ribas-Barba L, Serra-Majem L, Salvador G, et al. (2007) Trends in dietary habits and food consumption in Catalonia, Spain (1992-2003). Public Health Nutr 10, 1340-1353.

11. Marques-Vidal P, Ravasco P, Dias CM, et al. (2006) Trends of food intake in Portugal, 1987-1999: results from the National Health Surveys. Eur J Clin Nutr 60, 1414-1422.
12. WHO (1990) Diet, Nutrition and the Prevention of Chronic Disease. WHO Technical Report Series no. 916. Geneva: WHO.

13. WCRF, AICR (2007) Food, Nutrition, Physical Activity, And The Prevention of Cancer: A Global Perspective, 2nd ed. Washington, DC: AICR.

14. USDA (2005) Dietary Guidelines for Americans. Washington, DC: U.S. Department of Human Services.

15. MHLW, MAFF (2005) Japanese Food Guide Spinning Top. Toyko: Ministry of Health, Labour and Welfare and Ministry of Agriculture, Forestry and Fisheries. http://www.mhlw.go.jp/ bunya/kenkou/pdf/eiyou-syokuji5.pdf

16. KNS (2005) Dietary Reference Intakes for Koreans. Seoul: Korea Nutrition Society.

17. MHAW (2003) Healthy Plan 2010. Seoul: Ministry of Health and Welfare.

18. Eiben G, Andersson CS, Rothenberg E, et al. (2004) Secular trends in diet among elderly Swedes - cohort comparisons over three decades. Public Health Nutr 7, 637-644.

19. Krachler B, Eliasson MC, Johansson I, et al. (2005) Trends in food intakes in Swedish adults 1986-1999: findings from the Northern Sweden MONICA (Monitoring of Trends and Determinants in Cardiovascular Disease) Study. Public Health Nutr 8, 628-635.

20. Pollard C, Miller M, Woodman RJ, et al. (2009) Changes in knowledge, beliefs, and behaviors related to fruit and vegetable consumption among Western Australian adults from 1995 to 2004. Am J Public Health 99, 355-361.

21. Serdula MK, Gillespie C, Kettel-Khan L, et al. (2004) Trends in fruit and vegetable consumption among adults in the United States: behavioral risk factor surveillance system, 1994-2000. Am J Public Health 94, 1014-1018.

22. Kant AK, Graubard BI \& Kumanyika SK (2007) Trends in black-white differentials in dietary intakes of U.S. adults, 1971-2002. Am J Prev Med 32, 264-272.

23. Kranz S, Siega-Riz AM \& Herring AH (2004) Changes in diet quality of American preschoolers between 1977 and 1998. Am J Public Health 94, 1525-1530.

24. Johnson B \& Hackett AF (2007) Trends in fruit, vegetable and salad intakes in 9-10-year-old schoolchildren living in Liverpool, 2000-2005. Public Health Nutr 10, 252-255.

25. Nicklas TA, Demory-Luce D, Yang SJ, et al. (2004) Children's food consumption patterns have changed over two decades (1973-1994): the Bogalusa Heart Study. J Am Diet Assoc 104, 1127-1140.

26. Demory-Luce D, Morales M, Nicklas T, et al. (2004) Changes in food group consumption patterns from childhood to young adulthood: the Bogalusa Heart Study. J Am Diet Assoc 104, $1684-1691$.

27. Jungjohann SM, Luhrmann PM, Bender R, et al. (2005) Eight-year trends in food, energy and macronutrient intake in a sample of elderly German subjects. Br J Nutr 93, 361-378.

28. KHIDI (2006) The Third Korea National Health \& Nutrition Examination Survey(KNHANES III), 2005 - Nutrition Survey(I). Seoul: Korea Health Industry Development Institute $\&$ Ministry of Health and Welfare. 